\title{
ON ANALYSIS AND SYNTHESIS OPERATORS AND CHARACTERIZATION OF SYNTHESIS MATRIX OF A FRAME IN TERMS OF FRAME OPERATOR.
}

\author{
L. Njagi ${ }^{1}$, B.M. Nzimbi ${ }^{2}$ and S.K. Moindi ${ }^{3}$ \\ ${ }^{* 1}$ Department of Mathematics, Meru University of Science and Technology, P.O. Box 972- 60200, Meru. \\ ${ }^{2,3}$ School of Mathematics, University of Nairobi, Chiromo Campus, P. O. Box 30197-00100, Nairobi.
}

*Corresponding Author: -

E-mail:Injagi@must.ac.ke

\begin{abstract}
: -
In this research paper we introduce the operators associated with a frame. That is the Analysis and the Synthesis Operators and their basic properties. The structure of matrix representation of the Synthesis operator is also analysed. This matrix is what most frame constructions in fact focus on. The frame operator which is just the joining together of the analysis and synthesis operators is fundamental for the reconstruction of signals form frame coefficients. We also give a complete characterization of the synthesis matrix in terms of the frame operator.
\end{abstract}

Key words: Frame, analysis, synthesis, adjoint, Gramian, dual, operators. 


\subsection{INTRODUCTION}

We set $\ell^{M}=\ell_{2}(1 \ldots, M)$. This space in fact coincides with $\mathbb{R}^{m}$ or $\mathbb{C}^{m}$, endowed with the standard inner product and the associated Euclidean norm.

The analysis, synthesis, and frame operators determine the operations of a frame when analysing and reconstructing a signal. The Gramian operator is perhaps not that well known, yet it crucially illuminates the behaviour of a frame $\left(\varphi_{\mathrm{i}}\right)_{i=1}^{\mathcal{M}}$ embedded as an $\mathrm{N}$-dimensional subspace in the high-dimensional space $\ell^{m}$.

\subsection{Analysis and synthesis Operators}

Two of the main operators associated with a frame are the analysis and synthesis operators. The analysis operator - as the name suggests - analyses a signal in terms of the frame by computing its frame coefficients. We start by formalizing this notion.

Definition 1. Let $\left(\varphi_{i}\right)_{i=1}^{M}$ be a family of vectors in $\mathrm{H}^{\mathrm{N}}$. Then the associated analysis operator T:

$\mathrm{H}^{\mathrm{N}} \rightarrow \ell_{2}^{m}$ is define by

$$
\mathrm{T} x=\left(\left\langle x, \varphi_{i}\right\rangle\right), x \in \mathrm{H}^{\mathrm{N}} .
$$

In the following lemma we derive two basic properties of the analysis operator.

Lemma 1. Let $\left(\varphi_{i}\right)_{i=1}^{M}$ be a sequence of vectors in $\mathrm{H}^{\mathrm{N}}$ with associated analysis operator $\mathrm{T}$.

i) We have $\|\mathrm{T} x\|^{2}=\sum_{\mathrm{i}=1}^{\mathrm{m}}\left|\left\langle x, \varphi_{i}\right\rangle\right|^{2}$ for all $x \in \mathrm{H}^{\mathrm{N}}$.

Hence, $\left(\varphi_{i}\right)_{i=1}^{M}$ is a frame for $\mathrm{H}^{\mathrm{N}}$ if and only if $\mathrm{T}$ is injective.

ii) The adjoint operator $\mathrm{T}^{*}: \ell_{2}^{\mathrm{M}} \rightarrow \mathrm{H}^{\mathrm{N}}$ of $\mathrm{T}$ is given by

$$
\mathrm{T}^{*}\left(\varphi_{i}\right)_{\mathrm{i}=1}^{\mathrm{M}}=\sum_{\mathrm{i}=1}^{\mathrm{M}} a_{i} \varphi_{\mathrm{i}}
$$

Proof. (i) This is an immediate consequence of the definition of $\mathrm{T}$ and the frame property.

ii) For $x=\left(a_{i}\right)_{i=1}^{M}$ and $y \in \mathrm{H}^{\mathrm{N}}$, we have

$$
\left(\mathrm{T}^{*} x, y\right)=(x, \mathrm{~T} y)=\left\langle\left(a_{i}\right)_{i=1}^{M},\left(\left\langle y, \varphi_{i}\right\rangle\right)_{i=1}^{M}\right\rangle=\sum_{i=1}^{m} a_{i} \overline{\left\langle y, \varphi_{l}\right\rangle}=\left\langle\sum_{i=1}^{m} a_{i} \varphi_{i}, y\right\rangle .
$$

Thus, $\mathrm{T}^{*}$ is adjoint operator.

The second main operator associated to a frame, the synthesis operator, is now defined as the adjoint operator to the analysis operator given in Lemma1 (ii).

Definition 2. Let $\left(\varphi_{i}\right)_{i=1}^{M}$ be a sequence of vectors in $\mathrm{H}^{\mathrm{N}}$ with associated analysis operator $\mathrm{T}$. Then the associated synthesis operator is defined to be the adjoint operator $\mathrm{T}^{*}$.

The next result summarizes some basic, but useful, properties of the synthesis operator.

Lemma 2. Let $\left(\varphi_{i}\right)_{i=1}^{M}$ be a sequence of vectors in $\mathrm{H}^{\mathrm{N}}$ with associated analysis operator $\mathrm{T}$.

I. Let $\left(\mathrm{e}_{\mathrm{i}}\right)_{\mathrm{i}=1}^{\mathrm{M}}$ denote the standard basis of $\ell_{2}^{\mathrm{m}}$. Then for all $\mathrm{i}=1,2, \ldots, \mathrm{M}$, we have $\mathrm{T}^{*} \mathrm{e}_{\mathrm{i}}$

$=\mathrm{T}^{*} \mathrm{Pe}_{\mathrm{i}}=\varphi_{\mathrm{i}}$, where $\mathrm{P}: \ell_{2}^{\mathrm{m}} \rightarrow \ell_{2}^{\mathrm{m}}$ denotes the orthogonal projection onto $\mathrm{ran} T$.

II. $\quad\left(\varphi_{i}\right)_{i=1}^{M}$ is a frame if and only if $\mathrm{T}^{*}$ is surjective.

Proof. The first claim follows immediately from Lemmal and the fact that Ker $T^{*}=(\operatorname{ann} T)^{\perp}$. The second claim is a consequence of $\operatorname{ran} T^{*}=(\operatorname{ker} T)^{\perp}$ and Lemma 1(i).

Often frames are modified by the application of an invertible operator. The next result shows not only the impact on the associated analysis operator, but also the fact that the new sequence again forms a frame.

Proposition 3. Let $\varnothing=\left(\varphi_{i}\right)_{\mathrm{i}=1}^{\mathrm{M}}$ be a sequence of vectors in $\mathrm{H}^{\mathrm{N}}$ with associated analysis operator Tø and let $\mathrm{F}$ : $\mathrm{H}^{\mathrm{N}} \rightarrow \mathrm{H}^{\mathrm{N}}$ be a linear operator. Then the analysis operator of the sequence $F \emptyset=\left(F \varphi_{i}\right)_{i=1}^{M}$ is given by

$$
\mathrm{TF} \varnothing=\mathrm{T} \varnothing \mathrm{F}^{*} .
$$

Moreover, if $\emptyset$ is a frame of $\mathrm{H}^{\mathrm{N}}$ and $\mathrm{F}$ is invertible, then $\mathrm{F} \emptyset$ is also a frame for $\mathrm{H}^{\mathrm{N}}$.

Proof. For $x \in \mathrm{H}^{\mathrm{N}}$ we have

$$
\mathrm{TF} \emptyset x=\left(\left\langle x, \varphi_{\mathrm{i}}\right\rangle\right)_{\mathrm{i}=1}^{\mathrm{M}}=\left(\left\langle\mathrm{F}^{*} x, \varphi_{\mathrm{i}}\right\rangle\right)_{\mathrm{i}=1}^{\mathrm{M}} \mathrm{T} \emptyset \mathrm{F}^{*} x .
$$

This proves $\mathrm{TF} \emptyset=\mathrm{T} \varnothing \mathrm{F}^{*}$. The moreover part follows from lemma 2 (ii). 
Next, we analyse the structure of the matrix representation of the synthesis operator. This matrix is of fundamental importance, since this is what most frame constructions in fact focus on.

The first result provides the form of this matrix along with stability properties.

Lemma 4. $\emptyset=\left(\varphi_{i}\right)_{\mathrm{i}=1}^{\mathrm{M}}$ be a frame for $\mathrm{H}^{\mathrm{N}}$ with analysis operator $\mathrm{T}$, then a matrix representation of the synthesis operator $\mathrm{T}^{*}$ is the $\mathrm{N} \times \mathrm{M}$ matrix given by

$$
\left[\begin{array}{cccc}
\mid & \mid & \cdots & \mid \\
\varphi_{1} & \varphi_{2} & \cdots & \varphi_{m} \\
\mid & \mid & \cdots & \mid
\end{array}\right]
$$

Moreover, the riesz bounds of the row vectors of this matrix equal the frame bounds of the column vectors.

Proof. The form of the matrix representation is obvious. To prove the moreover part, let $\left(e_{j}\right)_{j=1}^{N}$ be the corresponding orthonormal basis of $\mathrm{H}^{\mathrm{N}}$ and for $\mathrm{j}=1,2, \ldots, \mathrm{N}$ Let

$$
\psi_{j}=\left[\left\langle\varphi_{1}, e_{j}\right\rangle,\left\langle\varphi_{2}, e_{j}\right\rangle, \ldots\left\langle\varphi_{m}, e_{j}\right\rangle\right]
$$

Be the row vectors of the matrix. Then for $x=\sum_{j=1}^{N} a_{j} e_{j}$ we obtain

$$
\begin{aligned}
\sum_{i=1}^{m}\left|\left\langle x, \varphi_{i}\right\rangle\right|^{2} & =\sum_{i=1}^{m} \sum_{j=1}^{N} a_{j}\left\langle e_{j}, \varphi_{i}\right\rangle=\sum_{j, k=1}^{N} a_{j} \overline{a_{k}} \sum_{i=1}^{M}\left\langle e_{j} \varphi_{i}\right\rangle\left\langle\varphi_{i}, e_{k}\right\rangle \\
& =\sum_{j, k=1}^{N} a_{j} \overline{a_{k}}\left\langle\psi_{k}, \psi_{j}\right\rangle=\left\|\sum_{j=1}^{N} \overline{a_{j}} \psi_{j}\right\|^{2} .
\end{aligned}
$$

A much stronger result (Proposition 9) can be proven for the case in which the matrix representation is derived using a specifically chosen orthonormal basis. However, the choice of this orthonormal basis requires the introduction of the frame operator in the following Section 2.

\subsection{The Frame Operator}

The frame operator may be considered the most important operator associated with a frame. Although it is "merely" the joining together of the analysis and synthesis operators, it encodes crucial properties of the frame. Moreover, it is also fundamental for the reconstruction of signals from frame coefficients.

\subsection{Fundamental Properties}

The precise definition of the frame operator associated with a frame is as follow:

Definition 3. Let $\left(\varphi_{i}\right)_{i=1}^{M}$ be a sequence of vectors in $\mathrm{H}^{\mathrm{N}}$ with associated analysis operator $\mathbf{T}$. Then the associated frame operator $\mathrm{S}: \mathrm{H}^{\mathrm{N}} \rightarrow \mathrm{H}^{\mathrm{N}}$ is defined by

$$
\mathrm{S} x=\mathrm{T}^{*} \mathrm{~T} x=\sum_{i=1}^{M}\left\langle x, \varphi_{i}\right\rangle \varphi_{i}, x \in H^{\mathrm{N}} .
$$

A first observation concerning the close relation of the frame operator to frame properties is the following lemma.

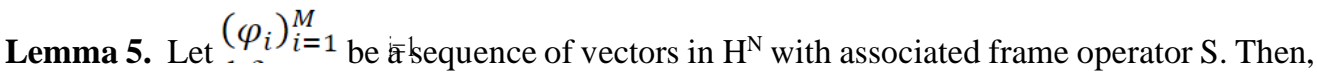

for all

$\mathrm{x} \in \mathrm{H}^{\mathrm{N}}$

$$
\langle S x, x\rangle=\sum_{i=1}^{M}\left|\left\langle x, \varphi_{i}\right\rangle\right|^{2} .
$$

Proof . The proof follows directly from $\langle S x, x\rangle=\langle T * T x, x\rangle=\|T x\|^{2}$ and Lemma 1(i)

Clearly, the frame operator $\mathrm{S}=\mathrm{T}^{*} \mathrm{~T}$ is self-adjoint and positive. The most fundamental property of the frame operator - if the underlying sequence of vectors forms a frame - is its invertibility, which is crucial for the reconstruction formula.

Theorem 6. The frame operator $\mathrm{S}$ of a frame $(\varphi i)_{i=1}^{M}$ for $\mathrm{H}^{\mathrm{N}}$ with frame bounds $\alpha$ and $\beta$ is a positive, self-adjoint invertible operator satisfying

$$
\alpha \text { Id } \leq \mathrm{S} \leq \beta \text {.Id }
$$

Proof. By Lemma 5, we have

$$
\langle\alpha x, x\rangle=\alpha\|x\|^{2} \leq \sum_{i=1}^{m}\left|\left\langle x, \varphi_{i}\right\rangle\right|^{2}=\langle S x, x\rangle \leq \beta\|x\|^{2}=\langle\beta x, x\rangle \text { for all } x \in \mathrm{H}^{\mathrm{N}} .
$$

This implies the claimed inequality.

The following proposition follows directly from proposition 3 
Proposition 7. Let $(\varphi i)_{i=1}^{M}$ be a frame for $\mathrm{H}^{\mathrm{N}}$ with frame operator $\mathrm{S}$, and let $\mathrm{F}$ be an invertible operator on $\mathrm{H}^{\mathrm{N}}$. Then $\left(\left(\mathrm{F} \varphi_{\mathrm{i}}\right)_{\mathrm{i}=1}^{\mathrm{M}}\right.$ is a frame with frame operator FSF*.

\subsection{The special case of tight frames}

Tight frames can be characterized as those frames whose frame operator equals a positive multiple of the identity. The next result provides a variety of similarly frame-operator-inspired classification.

Proposition 8. Let $(\varphi i)_{i=1}^{M}$ be a frame of $\mathrm{H}^{\mathrm{N}}$ with analysis operator $\mathrm{T}$ and frame operator S. Then the following conditions are equivalent.

(i) $\quad\left(\varphi_{i}\right)_{\mathrm{i}=1}^{\mathrm{M}}$ is an $\alpha$-tight frame for $\mathrm{H}^{\mathrm{N}}$.

(ii) $\mathrm{S}=\alpha$.Id.

(iii) For every $x \in \mathrm{H}^{\mathrm{N}}$,

$$
\mathrm{X}=\alpha^{-1} \cdot \sum_{\mathrm{i}=1}^{\mathrm{m}}\left\langle x, \varphi_{i}\right\rangle \varphi_{i} .
$$

(iv) For every $x \in \mathrm{H}^{\mathrm{N}}$,

$$
\alpha\|x\|^{2}=\sum_{\mathrm{i}=1}^{\mathrm{M}}\left|\left\langle x, \varphi_{i}\right\rangle\right|^{2} .
$$

(v) $\quad \mathrm{T} / \sqrt{\alpha}$ is an isometry.

Proof. (i) $\Leftrightarrow$ (ii) $\Leftrightarrow$ (iii) $\Leftrightarrow$ (iv) These are immediate from the definition of the frame operator and from Theorem 6 .

(ii) $\Leftrightarrow$ (v) This follows from the fact that $\mathrm{T} / \sqrt{\alpha}$ is an isometry if and only if $\mathrm{T} * \mathrm{~T}=\alpha$.Id.

A similar result for the special case of a Parseval frame can be easily deduced from Proposition 8 by setting $\alpha=1$.

\subsection{Structure of the synthesis matrix}

We now apply the previously derived results to obtain a complete characterization of the synthesis matrix of a frame in terms of the frame operator.

Proposition 9. Let $\mathrm{T}: \mathrm{H}^{\mathrm{N}} \rightarrow l_{2}^{\mathrm{M}}$ be a linear operator, Let $\left(e_{j}\right)_{\mathrm{j}=1}^{\mathrm{N}}$ be an orthonormal basis of $\mathrm{H}^{\mathrm{N}}$, and let $\left(e_{j}\right)_{\mathrm{j}=1}^{\mathrm{N}}$ be a sequence of positive numbers. By $\mathrm{A}$, denote the $\mathrm{N} \times \mathrm{M}$ matrix representation of $\mathrm{T}^{*}$ with respect to $\left(e_{j}\right)_{\mathrm{j}=1}^{\mathrm{N}}$ (and the standard basis $\left(e_{j}\right)_{\mathrm{j}=1}^{\mathrm{N}}$ of $\left.\ell_{2}^{\mathrm{M}}\right)$. Then the following conditions are equivalent.
(i) $\left(T^{*} e_{i}\right)_{\mathrm{i}=1}^{\mathrm{M}}$ forms a frame for $\mathrm{H}^{\mathrm{N}}$ whose frame oferator had eigenvectors $\left(e_{j}\right)_{\mathrm{j}=1}^{\mathrm{N}}$ and associated eigenvalues $\left(\lambda_{\mathrm{j}}\right)_{\mathrm{j}=1}^{\mathrm{N}}$.
(ii) The rows of $A$ are orthogonal, and the $\mathrm{j}^{\text {th }}$ row square sums to $\lambda \mathrm{j}$.
(iii) The columns of $\mathrm{A}$ form a frame for $\ell_{2}^{\mathrm{N}}$, and $\mathrm{AA}^{*}=\operatorname{diag}\left(\lambda_{1}, \cdots, \lambda_{\mathrm{N}}\right.$.

Proof. Let $\left(f_{j}\right)_{j=1}^{N}$ be the standard basis of $\ell^{N}$ and denote by $\mathrm{U}: \ell_{2}^{N} \rightarrow \mathrm{H}^{\mathrm{N}}$ the unitary operator which maps fj to ej. Then $T^{*}=U A$.

(i) $\Rightarrow$ (ii) Forj, $\mathrm{k} \in\{1, \cdots, \mathrm{N}\}$ wehave

$$
\left\langle A^{*} f_{j}, A^{*} f_{k}\right\rangle=\left\langle T U f_{j}, T U f_{k}\right\rangle=\left\langle T^{*} T e_{j}, e_{k}\right\rangle=\lambda_{j} \delta_{j k},
$$

Which is equivalent to (ii).

(ii) $\Rightarrow$ (iii) since the rows of $\mathrm{A}$ are orthogonal, we have rank $\mathrm{A}=\mathrm{N}$, which implies that the columns of $\mathrm{A}$ form a frame for $\ell^{N}$. The rest follows from $\left\langle A A^{*} f_{j}, f_{k}\right\rangle=\left\langle A^{*} f_{j}, A^{*} f_{k}\right\rangle=\lambda_{j} \delta_{j k}$ for $\mathrm{j}, \mathrm{k}=1, \cdots, \mathrm{N}$.

(iii) $\Rightarrow$ (i) since $\left(A \hat{\mathrm{e}}_{i}\right)_{i=1}^{M}$ is a spanning set for $\ell_{2}^{N}$ and $\mathrm{T}^{*}=\mathrm{UA}$, it follows that $\left(A \hat{\mathrm{e}}_{i}\right)_{i=1 \text { forms }}^{M}$ a frame for $\mathrm{H}^{\mathrm{N}}$. Its analysis operator is given by $\mathrm{T}$, since for all $x \in \mathrm{H}^{\mathrm{N}}$,

$$
\left(\left\langle x, T^{*} \hat{\mathrm{e}} i\right\rangle\right)_{i=1}^{M}=(\langle T x, \hat{\mathrm{e}} i\rangle)_{i=1}^{M}=\mathrm{Tx} .
$$

Moreover,

$$
\mathrm{T}^{*} T e_{j}=\mathrm{UAA} * \mathrm{U}^{*} \mathrm{e}_{\mathrm{j}}=\mathrm{U} \operatorname{diag}\left(\lambda_{1}, \cdots, \lambda_{N}\right) \mathrm{f}_{\mathrm{j}}=\lambda_{j} U f_{j}=\lambda_{j} e_{j},
$$

Which completes the proof.

\subsection{Gramian Operator}

Let $\left(\varphi_{i}\right)_{i=1}^{M}$ be a frame for $\mathrm{H}^{\mathrm{N}}$ with analysis operator $\mathrm{T}$. In the previous subsection we have looked at the properties of the frame operator defined by $\mathrm{S}=\mathrm{T} * \mathrm{~T}: \mathrm{H}^{\mathrm{N}} \rightarrow \mathrm{H}^{\mathrm{N}}$. Of particular interest is also the operator generated by first applying the synthesis and then the analysis operator. We first state the precise definition then discuss its importance. 
Definition 4. Let $\left(\varphi_{i}\right)_{i=1}^{M}$ be a frame of $\mathrm{H}^{\mathrm{N}}$ with analysis operator T. Then the operator $\mathrm{G}: \ell_{2}^{M} \rightarrow \ell_{2}^{M}$ defined by

$$
\mathrm{G}\left(a_{i}\right)_{i=1}^{M}=\mathrm{TT}^{*}\left(a_{i}\right)_{i=1}^{M}=\left(\sum_{i=1}^{M} a_{i}\left\langle\varphi_{i}, \varphi_{k}\right\rangle\right)_{k=1}^{M}=\sum_{i=1}^{M} a_{i}\left(\left\langle\varphi_{i}, \varphi_{k}\right\rangle\right)_{k=1}^{m}
$$

Is called the Gramian (operator) of the frame $\left(\varphi_{i}\right)$.

Note that the (canonical) matrix representation of the Gramian of a frame $\left(\varphi_{i}\right)_{i=1}^{M}$ for $\mathrm{H}^{\mathrm{N}}$ which will also be called the Gramian matrix) is given by

$$
\left[\begin{array}{cccc}
\left\|\varphi_{1}\right\|^{2} & \left\langle\varphi_{2}, \varphi_{1}\right\rangle & \cdots & \left\langle\varphi_{M}, \varphi_{1}\right\rangle \\
\left\langle\varphi_{1}, \varphi_{2}\right\rangle & \left\|\varphi_{2}\right\|^{2} & \cdots & \left\langle\varphi_{M}, \varphi_{2}\right\rangle \\
\vdots & \vdots & \ddots & \vdots \\
\left\langle\varphi_{1}, \varphi_{M}\right\rangle & \left\langle\varphi_{2}, \varphi_{M}\right\rangle & \cdots & \left\|\varphi_{M}\right\|^{2}
\end{array}\right] .
$$

One property of the Gramian is immediate. In fact, if the frame is unit norm, then the entries of the Gramian matrix are exactly the cosines of the angles between the frame vectors. Hence, for instance, if a frame is equiangular, then all offdiagonal entries of the Gramian matrix have the same modulus.

The fundamental properties of the Gramian operator are collected in the following theorem.

Theorem 10. Let $\left(\varphi_{i}\right)_{i=1}^{M}$ be a frame for $\mathrm{H}^{\mathrm{N}}$ with analysis operator T, frame operator $\mathrm{S}$, and Gramian operator $\mathrm{G}$. Then the following statements hold.

(i) An operator $U$ on $H^{N}$ is unitary if and only if the Gramian of $\left(U \varphi_{i}\right)_{i=1}^{\mathrm{M}}$ coincides with $\mathrm{G}$.

(ii) The nonzero eigenvalues of $\mathrm{G}$ and $\mathrm{S}$ coincide.

(iii) $\left(\varphi_{i}\right)_{i=1}^{M}$ is a Parseval frame if and only if $\mathrm{G}$ is an orthogonal projection of rank $\mathrm{N}$ (namely onto the range of T).

(iv) $\mathrm{G}$ is invertible if and only it $\mathrm{M}=\mathrm{N}$.

Proof. (i) This follows immediately from the fact that the entries of the Gramian matrix for $\left(\varphi_{i}\right)_{i=1}^{M}$ are of the form $\left\langle U \varphi_{i}\right.$ $\left.U \varphi_{j}\right\rangle$.

(ii) Since $\mathrm{TT}^{*}$ and $\mathrm{T} * \mathrm{~T}$ have the same nonzero eigenvalues, the same is true for $\mathrm{G}$ and $\mathrm{S}$.

(iii)It is immediate to prove that $\mathrm{G}$ is self-adjoint and has rank N. Since $\mathrm{T}$ is injective, $\mathrm{T}^{*}$ is surjective, and $\mathrm{G}^{2}=\left(\mathrm{TT}^{*}\right)\left(\mathrm{TT}^{*}\right)=\mathrm{T}\left(\mathrm{T}^{*} \mathrm{~T}\right) \mathrm{T}^{*}$,

It follows that $\mathrm{G}$ is an orthogonal projection if and only if $\mathrm{T}^{*} \mathrm{~T}=\mathrm{Id}$, which is equivalent to the frame being Parseval.

(iv) This is immediate by (ii).

\subsection{Reconstruction from Frame Coefficients}

The analysis of a signal is typically performed by merely considering its frame coefficients. However, if the task is transmission of a signal, the ability to reconstruct the signal from its frame coefficients and also to do so efficiently becomes crucial. However, reconstruction from coefficients with respect to a redundant system is much more delicate and requires the utilization of another frame, called the dual frame. If computing such a dual frame is computationally too complex, a circumvention of this problem is the frame algorithm.

\subsection{Exact Reconstruction}

We start by stating an exact reconstruction formula.

Theorem 11. Let $\left(\varphi_{i}\right)_{i=1}^{M}$ be a frame for $\mathrm{H}^{\mathrm{N}}$ with frame operator $\mathrm{S}$. then, for every $x \in \mathrm{H}^{\mathrm{N}}$, we have

$$
x=\sum_{\mathrm{i}=1}^{\mathrm{M}}\left\langle x, \varphi_{\mathrm{i}}\right\rangle^{\mathrm{S}^{-1}} \varphi_{\mathrm{i}}=\sum_{\mathrm{i}=1}^{\mathrm{M}}\left\langle x, \mathrm{~S}^{-1} \varphi_{\mathrm{i}}\right\rangle \varphi_{\mathrm{i}}
$$

Proof. The proof follows directly from the definition of the frame operator in Definition 3 by writing $x=\mathrm{S}^{-1} \mathrm{~S} x$ and $x=\mathrm{SS}^{-1} x$.

Notice that the first formula can be interpreted as a reconstruction strategy, whereas the second formula has the flavour of a decomposition. We further observe that the sequence $\left(S^{-1} \varphi i\right)_{i=1}^{M}$ plays a crucial role in the formulas in Theorem 11 . The next result shows that this sequence indeed also constitutes a frame.

Proposition 12. Let $\left(\varphi_{i}\right)_{i=1}^{M}$ be a frame for $\mathrm{H}^{\mathrm{N}}$ with frame bounds $\alpha$ and $\beta$ and with frame operator $\mathrm{S}$. Then the sequence $\left(S^{-1} \varphi i\right)_{i=1}^{M}$ is a frame for $\mathrm{H}^{\mathrm{N}}$ with frame bound $\beta^{-1}$ and $\alpha^{-1}$ and with frame operator $\mathrm{S}^{-1}$.

Proof. By Proposition 7, the sequence $\left(S^{-1} \varphi i\right)_{i=1}^{M}$ forms a frame for $\mathrm{H}^{\mathrm{N}}$ with associated frame operator $\mathrm{S}^{-1}\left(\mathrm{~S}^{-1}\right)^{*}=\mathrm{S}^{-1}$. This in turn yields the frame bounds $\beta^{-1}$ and $\alpha^{-1}$. 
This new frame is called the canonical dual frame. In the sequel, we will discuss that other dual frame may also be utilized for reconstruction.

Definition 5. Let $\left(\varphi_{i}\right)_{i=1}^{M}$ be a frame for $\mathrm{H}^{\mathrm{N}}$ with framé operator denoted by $\mathrm{S}$. then $\left(S^{-1} \varphi i\right)_{i=1}^{M}$ is called the canonical dual frame for $\left(\varphi_{i}\right)_{i=1}^{M}$.

The canonical dual frame of a Perseval frame is now easily determined by Proposition 12.

Corollary 13. Let $\left(\varphi_{i}\right)_{i=1}^{M}$ be a Parseval frame for $\mathrm{H}^{\mathrm{N}}$. Then its canonical dual frame is the frame $\left(\varphi_{i}\right)_{i=1}^{M}$ itself, and the reconstruction formula in Theorem 11 reads

$$
x=\sum_{i=1}^{M}\left\langle x, \varphi_{i} \varphi_{1}\right\rangle, x \in \mathrm{H}^{\mathrm{N}}
$$

As an application of the above reconstruction formula for Parseval frames, we prove the following proposition which again shows the close relation between Parseval frames and orthonormal bases.

Proposition 14. (Trace Formula for Parseval Frames) Let $\left(\varphi_{i}\right)_{i=1}^{M}$ be a Parseval frame for $\mathrm{H}^{\mathrm{N}}$, and let $\mathrm{F}$ be a linear operator on $\mathrm{H}^{\mathrm{N}}$. Then

$$
\operatorname{Tr}(\mathrm{F})=\sum_{i=1}^{M}\left\langle F \varphi_{\mathrm{i}}, \varphi_{\mathrm{i}}\right\rangle
$$

Proof. Let $\left(e_{j}\right)_{j=1}^{N}$ be on orthonormal basis for $\mathrm{H}^{\mathrm{N}}$. Then, by definition.

$$
\operatorname{Tr}(\mathrm{F})=\sum_{i=1}^{M}\left\langle F e_{j}, e_{j}\right\rangle \text {. }
$$

This implies

$$
\begin{aligned}
& \operatorname{Tr}(\mathrm{F})=\sum_{j=1}^{N}\left\langle\sum_{i=1}^{M}\left\langle F e_{j}, \varphi_{i}\right\rangle \varphi_{\mathrm{i}}, \varphi_{\mathrm{j}}\right\rangle=\sum_{j=1}^{N} \sum_{i=1}^{M}\left\langle e_{j}, F^{*} \varphi_{\mathrm{i}}\right\rangle\left\langle\varphi_{\mathrm{i}}, e_{j}\right\rangle \\
& =\sum_{i=1}^{M}\left\langle\sum_{j=1}^{N}\left\langle\varphi_{\mathrm{i}}, e_{j}\right\rangle e_{j}, F^{*} \varphi_{\mathrm{i}}\right\rangle=\sum_{i=1}^{M}\left\langle\varphi_{\mathrm{i}}, F^{*} \varphi_{\mathrm{i}}\right\rangle=\sum_{i=1}^{M}\left\langle F \varphi_{\mathrm{i}}, \varphi_{\mathrm{i}}\right\rangle .
\end{aligned}
$$

As mentioned earlier, many other dual frames for reconstruction exist. We now give a precise definition of the same.

Definition 6. Let $\left(\varphi_{i}\right)_{i=1}^{M}$ be a frame for $\mathrm{H}^{\mathrm{N}}$. Then a frame $\left(\mathrm{\Psi}_{\mathrm{i}}\right)_{i=1}^{m}$ is called dual frame for $\left(\varphi_{i}\right)_{i=1}^{M}$, if

$$
x=\sum_{i=1}^{M}\left\langle x, \varphi_{\mathrm{i}}\right\rangle \Psi_{\mathrm{i}} \text { for all } x \in \mathrm{H}^{\mathrm{N}} \text {. }
$$

Dual frames, which do not coincide with the canonical dual frame, are often coined alternate dual frames.

Similar to the different forms of the reconstruction formula in Theorem 11, dual frames can also achieve reconstruction in different ways.

Proposition 15. Let $\left(\varphi_{i}\right)_{i=1}^{M}$ and $\Psi_{\mathrm{i}_{\mathrm{i}=1}}^{\mathrm{M}}$ be frames for $\mathrm{H}^{\mathrm{N}}$ and let $\mathrm{T}$ and $\widetilde{\mathrm{T}}_{\text {be the analysis operators of }}\left(\varphi_{i}\right)_{i=1}^{M}$ and $\mathrm{\Psi}_{\mathrm{i}_{\mathrm{i}=1}^{\mathrm{M}}}^{\mathrm{M}}$, respectively. Then the following conditions are equivalent.

(i) We have $x=\sum_{\mathrm{i}=1}^{\mathrm{M}}\left\langle x, \Psi_{\mathrm{i}}\right\rangle \varphi \mathrm{i}$ for all $x \in \mathrm{H}^{\mathrm{N}}$.

(ii) We have $x=\sum_{\mathrm{i}=1}^{\mathrm{M}}\left\langle x, \varphi_{\mathrm{i}}\right\rangle \Psi_{\mathrm{i}}$ for all $x \in \mathrm{H}^{\mathrm{N}}$.

(iii) We have $(x, y)=\sum_{\mathrm{i}=1}^{\mathrm{M}}\left\langle x, \varphi_{\mathrm{i}}\right\rangle\langle\psi \mathrm{i}, y\rangle$ for all $x, y \in \mathrm{H}^{\mathrm{N}}$.

(iv) $\quad \mathrm{T}^{*} \widetilde{\mathrm{T}}=\mathrm{Id}$ and $\widetilde{\mathrm{T}} * \mathrm{~T}=\mathrm{Id}$.

Proof. Clearly (i) is equivalent to $\mathrm{T}^{*} \widetilde{\mathrm{T}}=\mathrm{Id}$, which holds if and only if $\widetilde{\mathrm{T}}^{*} \mathrm{~T}=\mathrm{Id}$. The equivalence of (iii) can be derived in a similar way.

One may ask what distinguishes the canonical dual frame from the alternate dual frames besides its explicit formula in terms of the initial frame. Another seemingly different question is which properties of the coefficient sequence in the composition of some signal $x$ in terms of the frame (see Theorem 11).

$$
x=\sum_{i=1}^{M}\left\langle x, S^{-1} \varphi_{\mathrm{i}}\right\rangle \varphi_{\mathrm{i}}
$$

uniquely distinguishes it from other coefficient sequences; redundancy allows infinitely many coefficient sequences. Interestingly, the next result answers both questions simultaneously by stating that this coefficient sequence has minimal $\ell_{2}$-norm among all sequences - in particular those, with respect to alternate dual frames - representing $x$.

Proposition 16. Let $\left(\varphi_{i}\right)_{i=1}^{M}$ be a frame for $\mathrm{H}^{\mathrm{N}}$ with frame operator $\mathrm{S}$, and let $x \in \mathrm{H}^{\mathrm{N}}$. If $\left(\mathrm{a}_{\mathrm{i}}\right)_{\mathrm{i}=1}^{\mathrm{M}}$ are scalars such that $x=$ $\sum_{\mathrm{i}=1}^{\mathrm{M}} \mathrm{a}_{\mathrm{i}} \varphi_{\mathrm{i}}$, then 
Proof. Letting $\mathrm{T}$ denote the analysis operator of $\left(\varphi_{\mathrm{i}}\right)_{i=1}^{M}$, we obtain

$$
\sum_{\mathrm{i}=1}^{\mathrm{M}}\left|\mathrm{a}_{\mathrm{i}}\right|^{2}=\sum_{\mathrm{i}=1}^{\mathrm{M}}\left|\left\langle x, \mathrm{~S}^{-1} \varphi_{\mathrm{i}}\right\rangle\right|^{2}+\sum_{\mathrm{i}=1}^{\mathrm{M}}\left|\mathrm{a}_{\mathrm{i}}-\left\langle x, \mathrm{~S}^{-1} \varphi_{\mathrm{i}}\right\rangle\right|^{2} .
$$

$$
\left(\left\langle x, S^{-1} \varphi_{\mathrm{i}}\right\rangle\right)_{i=1}^{M}=\left(\left\langle S^{-1} x, \varphi_{\mathrm{i}}\right\rangle\right)_{i=1}^{M} \in \operatorname{ran} \mathrm{T} .
$$

Since $x=\sum_{i=1}^{M} a_{i} \varphi_{\mathrm{i}}$, it follows that

$$
\left(a_{i}-\left\langle x, S^{-1} \varphi_{\mathrm{i}}\right\rangle\right)_{i=1}^{M} \in \operatorname{ker} \mathrm{T}^{*}=(\operatorname{ran} \mathrm{T}) \perp .
$$

Considering the decomposition

$$
\left(a_{i}\right)_{i=1}^{M}=\left(\left\langle x, S^{-1} \varphi_{\mathrm{i}}\right\rangle\right)_{i=1}^{M}+\left(a_{i}-\left\langle x, S^{-1} \varphi_{\mathrm{i}}\right\rangle\right)_{i=1}^{M},
$$

The claim is immediate.

Corollary 17. Let $\left(\varphi_{i}\right)_{i=1}^{M}$ be a frame for $\mathrm{H}^{\mathrm{N}}$, and let $\left(\Psi_{\mathrm{i}}\right)_{\mathrm{i}=1}^{\mathrm{M}}$ be an associated alternate dual frame. Then, for all $x$ $\in \mathrm{H}^{\mathrm{N}}$,

$$
\left\|\left(\left\langle x, \mathrm{~S}^{-1} \varphi_{\mathrm{i}}\right\rangle\right)_{\mathrm{i}=1}^{\mathrm{M}}\right\|^{2} \leq\left\|\left(\left\langle x, \Psi_{\mathrm{i}}\right\rangle\right)_{\mathrm{i}=1}^{\mathrm{M}}\right\|^{2} .
$$

\subsection{Properties of Dual Frames}

While we focused on properties of the canonical dual frame in the last subsection, we now discuss properties shared by all dual frames. The first question that arises is:

How do we characterize all dual frames? And a comprehensive answer is provided by the following result.

Proposition 18. Let $\left(\varphi_{i}\right)_{i=1}^{M}$ be a frame for $\mathrm{H}^{\mathrm{N}}$ with analysis operator $\mathrm{T}$ and frame operator $\mathrm{S}$. Then the following conditions are equivalent.

(i) $\quad\left(\Psi_{\mathrm{i}}\right)_{\mathrm{i}=1}^{\mathrm{M}}$ is a dual frame for $\left(\varphi_{\mathrm{i}}\right)_{\mathrm{i}=1}^{\mathrm{M}}$.

(ii) The analysis operator $\mathrm{T}_{1}$ of the sequence $\left(\Psi_{\mathrm{i}}-\mathrm{S}^{-1} \varphi_{\mathrm{i}}\right)_{\mathrm{i}=1}^{\mathrm{M}}$ satisfies $\operatorname{Ran} \mathrm{T} \perp \operatorname{ran} \mathrm{T}_{1}$.

Proof. We set $\widetilde{\Psi_{1}}:=\Psi_{\mathrm{i}}-\mathrm{S}^{-1} \varphi_{\mathrm{i}}$ for $\mathrm{I}=1, \cdots, \mathrm{M}$ and note that

$$
\sum_{i=1}^{M}\left\langle x, \Psi_{\mathrm{i}}\right\rangle \varphi_{\mathrm{i}}=\sum_{i=1}^{M}\left\langle x, \tilde{\varphi} i+S^{-1} \varphi_{\mathrm{i}}\right\rangle \varphi_{\mathrm{i}}=x+\sum_{i=1}^{M}\langle x, \tilde{\varphi} i\rangle \varphi_{\mathrm{i}}=x+\mathrm{T}^{*} \mathrm{~T}_{1} x
$$

holds for all $x \in \mathrm{H}^{\mathrm{N}}$. Hence $\left(\Psi_{\mathrm{i}}\right)_{i=1}^{M}$ is a dual frame for $\left(\varphi_{i}\right)_{i=1}^{M}$ if and only if $\mathrm{T} * \mathrm{~T} 1=0$. But this is equivalent to (ii). $i=1 i=1$

From this result, we have the following corollary which provides a general formula for all dual frames.

Corollary 19. Let $\left(\varphi_{i}\right)_{i=1}^{M}$ be a frame for $\mathrm{H}^{\mathrm{N}}$ with analysis operator $\mathrm{T}$ and frame operator $\mathrm{S}$ with associated normalized eigenvectors $\left(e_{j}\right)_{\mathrm{j}=1}^{\mathrm{N}}$ and respective eigenvalues $\left(\lambda_{j}\right)_{j=1}^{\mathrm{N}}$. Then every dual frame $\left(\psi_{\mathrm{i}}\right)_{\mathrm{i}=1}^{\mathrm{M}}$ for $\left(\varphi_{\mathrm{i}}\right)_{\mathrm{i}=1}^{\mathrm{M}}$ is of the form $j=1$

$$
\psi_{\mathrm{i}}=\sum_{\mathrm{j}=1}^{\mathrm{N}}\left(\frac{1}{\lambda_{\mathrm{j}}}\left\langle\varphi_{\mathrm{i}}, \mathrm{e}_{\mathrm{j}}\right\rangle+\overline{\mathrm{h}_{\mathrm{l}}}\right) \mathrm{e}_{\mathrm{j}}, \quad \mathrm{i}=1, \ldots, \mathrm{M},
$$

Where each $\left(\mathrm{h}_{\mathrm{ij}}\right)_{\mathrm{i}=1}^{\mathrm{M}}, \mathrm{j}=1, \ldots, \mathrm{N}$, is an element of $(\operatorname{ranT})^{\perp}$.

$$
\begin{aligned}
& M \\
& M
\end{aligned}\left(h_{i j}\right)_{i=1}^{M} \in \ell_{2}^{M}, \mathrm{j}=1, \ldots, \mathrm{N},{ }_{\text {then }} \psi_{\mathrm{i}}=\mathrm{S}^{-1} \varphi_{\mathrm{i}}+\tilde{\varphi} \mathrm{i} \text {, }
$$

Proof. If $\psi, \mathrm{i}=1, \ldots, \mathrm{M}, \mathrm{i}$ of the given form with sequences $\left(h_{i j}\right)_{i=1} \in \ell_{2}^{M}, \mathrm{j}=1, \ldots, \mathrm{N}$, then $\psi_{\mathrm{i}}=\mathrm{S}^{-1} \varphi_{\mathrm{i}}+\tilde{\varphi} \mathrm{i}$,
where $\tilde{\varphi} \mathrm{i}=\sum_{j=1}^{N} h_{l j} e_{j}, \mathrm{i}=1, \ldots, \mathrm{M}$. The analysis operator $\tilde{T}$ of $(\tilde{\varphi} i)_{i=1 \text { satisfies }}^{M} \tilde{T} \mathrm{e}_{\mathrm{j}}=\left(h_{i j}\right)_{i=1}^{M}$ The claim follows from this observation.

\subsection{Conclusion}

We have discussed the analysis, synthesis and frame operators of a frame in a finite dimensional Hilbert space. We have also looked at their basic properties and the structure of matrix representation of the synthesis operator, where the Riesz bounds of the row vectors of the matrix equal the frame bounds of the column vectors. The frame operator is considered the most important operator associated with a frame, although it is just the joining of the analysis and the synthesis operators. It encodes crucial properties of the frame as well as it is fundamental for the reconstruction of signals from frame coefficients. The frame operator $\mathrm{S}=\mathrm{T}^{*} \mathrm{~T}$ is self-adjoint and positive. If the underlying sequence of vectors forms a frame, then the frame operator is invertible, which is crucial for the reconstruction formula, given in Theorem 11. Properties of Dual frames were also discussed as can be seen in Proposition 18 and corollary 19. 


\subsection{References}

[1].Bodmann, B.G.: Optimal linear transmission by loss-insensitive packet encoding. Appl. Comput. Harmon. (2007)

[2].Bodmann, B., Casazza, P.G.: The road to equal-norm Parseval frames. J. Funct. (2010)

[3].Bodmann, B.G., Casazza, P.G., Kutyniok, G.: A quantitative notion of redundancy for finite frames. Appl. Comput. Harmon. (2011)

[4].Bodmann, B.G., Casazza, P.G., Paulsen, V.I., Speegle, D.: Spanning and independence properties of frame partitions. Proc. Am. Math. Soc. (2012)

[5].Bodmann, B., Lipshitz, S.: Randomly dithered quantization and sigma-delta noise shaping for finite frames. Appl. Comput. Harmon. (2008)

[6].Bodmann, B.G., Paulsen, V.I.: Frames, graphs and erasures. Linear Algebra Appl. (2005) 